\title{
Visuo-haptic transfer for object recognition in children with periventricular leukomalacia and bilateral cerebral palsy
}

\section{Giulia Purpura, Silvia Perazza, Giovanni Cioni \& Francesca Tinelli}

To cite this article: Giulia Purpura, Silvia Perazza, Giovanni Cioni \& Francesca Tinelli (2019):

Visuo-haptic transfer for object recognition in children with periventricular leukomalacia and bilateral cerebral palsy, Child Neuropsychology, DOI: 10.1080/09297049.2019.1602599

To link to this article: https://doi.org/10.1080/09297049.2019.1602599

Published online: 24 Apr 2019.

Submit your article to this journal $\sqsubset$

Wll Article views: 19

View Crossmark data \ulcorner 


\title{
Visuo-haptic transfer for object recognition in children with periventricular leukomalacia and bilateral cerebral palsy
}

\author{
Giulia Purpuraa, Silvia Perazza ${ }^{a}$, Giovanni Cioni ${ }^{a, b}$ and Francesca Tinelli ${ }^{\mathrm{a}}$
}

aDepartment of Developmental Neuroscience, IRCCS Stella Maris Foundation, Pisa, Italy; ${ }^{b}$ Department of Clinical and Experimental Medicine, University of Pisa, Pisa, Italy

\begin{abstract}
Object recognition is a complex adaptive process that can be impaired in children with neurodevelopmental disabilities. Recently, we found a significant effect of time on the development of unimodal and crossmodal recognition skills for common objects in typical children and this was a starting point for the study of visuo-haptic object recognition skills in impaired populations. In this study, we investigated unimodal visual information, unimodal haptic information and visuo-haptic information transfer in 30 children, from 4.0 to 10.11 years of age, with bilateral Periventricular Leukomalacia (PVL) and bilateral cerebral palsy. Results were matched with those of 116 controls. Participants were tested using a clinical protocol, adopted in the previous study, involving visual exploration of black-and-white photographs of common objects, haptic exploration of real objects and visuo-haptic transfer of these two types of information. Results show that in the PVL group as in controls, there is an agedependent development of object recognition abilities for visual, haptic and visuo-haptic modalities, even if PVL children perform worse in all the three conditions, in comparison with the typical group. Furthermore, PVL children have a specific deficit both in visual and haptic information processing, that improves with age, probably thanks to everyday experience, but the visual modality shows a better and more rapid maturation, remaining more salient compared to the haptic one. However, multisensory processes partially facilitate recognition of common objects also in PVL children and this finding could be useful for planning early intervention in children with brain lesion.
\end{abstract}

\section{ARTICLE HISTORY}

Received 24 September 2018 Accepted 24 March 2019

\section{KEYWORDS}

Visuo-haptic transfer; multisensory processing; object recognition; cerebral palsy; periventricular leukomalacia

Sensory processing and integration are crucial for learning and adaptation to the environment. Specifically, multisensory capabilities for object recognition require a long and complex period of maturation during childhood and are dependent on the progressive improvement of both unisensory channels and brain networks which are able to integrate different sensory information into a single and more accurate percept.

In particular, visual and haptic modalities are deeply interconnected in object processing, because these two sources of information show many similarities in terms of categorization, recognition, and representation, which suggest a common neural 
substrate and a close cooperation that builds on previous experiences with objects and guides decision making and behavioral responses in everyday life (Lacey \& Sathian, 2014). Lateral occipital complex (LOC) has been found to be specifically involved in early visuo-haptic recognition, and its development seems more protracted compared to unisensory neurological substrates (Joanne Jao, James, \& Harman James, 2014). This finding is in line with previous psychophysical data on visuo-haptic mechanisms, which suggest that integration of visual and haptic information becomes statistically optimal, adultlike, after the $8^{\text {th }}$ year of life (Gori, Del Viva, Sandini, \& Burr, 2008). In fact, according to Gori and colleagues, visuo-haptic processes are immature in young children and, before the $8^{\text {th }}$ year of life, one sense dominates the other, depending on the task. For example, for size discrimination, haptic information dominates in determining height and thresholds, while for orientation discrimination, visual information is more salient.

In our recent study (Purpura, Cioni, \& Tinelli, 2018), visuo-haptic object recognition was investigated in typical pre-school and school-aged children, and the findings confirmed the long developmental trend of multisensory transfer abilities. As a matter of fact, the development of visuo-haptic processes necessary for object recognition is typically protracted for the whole period of primary school, although children as young as 4-5 years of age are already partially able to benefit from this ability (Purpura et al., 2018). These results are in line with findings by Jovanovic \& Drewing (Jovanovic \& Drewing, 2014) who affirm that children integrate seemingly corresponding visuo-haptic information in similar ways as adults do, although these authors suggest that the children focus on a single sense, when information from different senses is strongly discrepant.

Important observations have been presented in the scientific literature about the typical development of multisensory processes, however, less is known about the interaction between visual and haptic information in children with neurodevelopmental disabilities. In particular, as far as object processing is concerned, non-sighted children seem to show a very poor haptic perception in orientation discrimination, but not in object recognition or in size identification (Gori, Sandini, Martinoli, \& Burr, 2010; Morrongiello, Humphrey, Timney, Choi, \& Rocca, 1994), while children with motor deficit present more evident difficulties in haptic and visual size discrimination (Gori, Tinelli, Sandini, Cioni, \& Burr, 2012) as well as in haptic object or shape recognition (Wingert, Burton, Sinclair, Brunstrom, \& Damiano, 2008). These findings support the idea that early cross-sensory calibration, which is necessary for the normal development of perceptual systems, can mature in a different manner when early sensory or motor disorders are present. However, developmental differences between unimodal and multimodal experiences have not yet been specifically investigated in these populations.

Continuing in this line of research, our principal aim was to study visuo-haptic transfer in two different groups of participants: the former with motor impairment i.e. with less experience in manipulating objects with his hands, the latter with important visual disorders i.e. with a very developed tactile capacity. In the current study, we report results about the first group, and for this reason, we analyzed unisensory and multisensory processing for object recognition in children with bilateral Periventricular Leukomalacia (PVL). This disorder consists in hypoxic-ischemic damage to periventricular white matter, which usually occurs at the beginning of the third trimester of 
gestation (Volpe, 2003). Depending on the extension of the lesion, children can develop motor disorders, usually bilateral cerebral palsy, with a primary deficit of the lower limbs, (Bax, Tydeman, \& Flodmark, 2006), and a wide spectrum of neurovisual symptoms, including object recognition disorders (Fazzi et al., 2009). We decided to enroll PVL children because there is evidence that cystic PVL correlates with a motor impairment (Volpe, 2009) involving the lower limbs, and when it is more extensive, the upper ones too. Furthermore, PVL population can also have difficulties in visual recognition of objects, so this population is suitable for understanding how brain networks and early environmental experiences interact to permit the re-organization of sensory calibration.

\section{Materials and methods}

\section{Participants}

Thirty children (M: 21, F: 9) were recruited from the Vision Laboratory of the IRCCS Stella Maris Foundation (Pisa, Italy), between 2015 and 2018, on the basis of the following criteria: i) presence of PVL at MRI, ii) diagnosis of bilateral cerebral palsy based on neurological examination and categorized using the Gross Motor Function Classification System (GMFCS) (Palisano et al., 1997) and the Manual Ability Classification System (MACS) (Eliasson et al., 2006), iii) verbal IQ> 70 measured with the Wechsler Scales, iv) age raging between 4 years and 10 years and 11 months, v) visual acuity $\geq 4 / 10$ (corresponding to normal or mildly reduced visual acuity, not consistent with severe visual impairment). As regards the GMFCS, motor impairment was classified according to five motor levels: Level I) walks without restrictions: limitations in more advanced gross motor skills; Level II) walks without an assistive device: limitations walking outdoors and in the community; Level III) walks with an assistive mobility device: limitations walking outdoors and in the community; Level IV) selfmobility with limitations; children are transported or use power mobility devices outdoors and in the community; Level V) self-mobility is severely limited notwithstanding the use of assistive technology (Palisano et al., 1997). As regards the MACS, impairment in manual ability was classified according to five levels:: Level I) handles objects easily and successfully; Level II) handles most objects but somewhat reduced quality and/or speed of achievement; Level III) handles objects with difficulty, needs help to prepare and/or modify activities; Level IV) handles a limited selection of easily managed objects in adapted situations; Level V) does not handle objects and has severely limited ability to perform even simple actions. Finally, as regards cognitive levels, the exact quotient of 4 PVL children was not available because the Weschler scales were administered in another hospital, however, their medical records confirmed a verbal quotient within the normal range. The characteristics of the whole PVL cohort are reported in Table 1.

The typical and term-born control sample (TD) was composed of 116 children (M: 64; F: 52), selected from a kindergarten and a primary school in Pisa (Italy). Children ranged in age from 4 years to 10 years and 11 months. No child enrolled in the study had major ophthalmological or neuropsychiatric disorders. All children had an intelligence quotient $>25^{\text {th }}$ percentile on Raven's Progressive Matrices (RPM). In children 4-6 years old we also evaluated the ability to name a series of objects and animals by 
Table 1. The distribution of PVL children among age groups.

\begin{tabular}{|c|c|c|c|c|c|c|c|c|c|c|}
\hline & $\begin{array}{l}\text { Participants } \\
\text { (n) }\end{array}$ & $\begin{array}{c}\text { Sex } \\
(\mathrm{M} / \mathrm{F})\end{array}$ & $\begin{array}{c}\text { Age } \\
\text { (ys, } \\
\text { ms) }\end{array}$ & GA & $\begin{array}{l}\text { Birth } \\
\text { Weight } \\
\text { (gr) }\end{array}$ & $\begin{array}{c}\text { Verbal } \\
\text { Quotients }\end{array}$ & $\begin{array}{l}\text { GMFCS } \\
\text { levels }\end{array}$ & $\begin{array}{l}\text { MACS } \\
\text { levels }\end{array}$ & $\begin{array}{l}\text { Visual } \\
\text { Acuity }\end{array}$ & Class school \\
\hline \multicolumn{11}{|l|}{ Group } \\
\hline \multicolumn{11}{|l|}{$A$} \\
\hline & 1 & $\mathrm{~F}$ & 4,3 & 40 & 3990 & 110 & IV & III & $10 / 10$ & II kindergarten class \\
\hline & 2 & $\mathrm{~F}$ & 5,9 & 29 & 1200 & NA & II & I & $10 / 10$ & III kindergarten class \\
\hline & 3 & $\mathrm{~F}$ & 4,9 & 38 & 3030 & 100 & $\|$ & $\|$ & $9 / 10$ & II kindergarten class \\
\hline & 4 & $M$ & 4,7 & 33 & NA & 95 & $\|$ & ॥ & $7 / 10$ & II kindergarten class \\
\hline & 5 & M & 4,9 & 32 & 1870 & 116 & $\|$ & III & $7 / 10$ & II kindergarten class \\
\hline & 6 & M & 5 & 28 & 1330 & 94 & $\|$ & III & $8 / 10$ & III kindergarten class \\
\hline & 7 & $M$ & 4,2 & 33 & 2060 & 92 & I & ॥ & $10 / 10$ & II kindergarten class \\
\hline & 8 & M & 6,3 & 28 & NA & 103 & IV & IV & $7 / 10$ & III kindergarten class \\
\hline & 9 & $M$ & 6,4 & 29 & 1000 & 106 & I & I & $8 / 10$ & III kindergarten class \\
\hline \multicolumn{11}{|l|}{ Group } \\
\hline \multicolumn{11}{|l|}{ B } \\
\hline & 10 & $M$ & 7,2 & 24 & 784 & 92 & III & III & $10 / 10$ & I primary school class \\
\hline & 11 & M & 9 & 28 & NA & 88 & $\|$ & I & $10 / 10$ & III primary school class \\
\hline & 12 & M & 8,11 & 31 & 1860 & 79 & III & III & $9 / 10$ & III primary school class \\
\hline & 13 & M & 7,3 & 31 & 1810 & 108 & III & III & $10 / 10$ & II primary school class \\
\hline & 14 & $\mathrm{~F}$ & 7,3 & 31 & 1580 & 104 & I & ॥ & $10 / 10$ & II primary school class \\
\hline & 15 & M & 7,11 & 33 & 1790 & 92 & i & I & $6 / 10$ & Il primary school class \\
\hline & 16 & M & 7,11 & 33 & 1940 & 72 & I & I & $5 / 10$ & Il primary school class \\
\hline & 17 & M & 7,11 & 27 & 970 & 116 & $\|$ & III & $10 / 10$ & Il primary school class \\
\hline & 18 & $\mathrm{~F}$ & 6 & 32 & NA & 112 & $\|$ & III & $10 / 10$ & I primary school class \\
\hline & 19 & M & 6,6 & 35 & 2250 & 92 & III & III & $5 / 10$ & I primary school class \\
\hline & 20 & $\mathrm{~F}$ & 8,8 & 29 & 1320 & 78 & IV & IV & $4 / 10$ & II primary school class \\
\hline & 21 & $M$ & 7 & 28 & 1216 & NA & I & ॥ & $10 / 10$ & I primary school class \\
\hline & 22 & $M$ & 10,2 & 28 & NA & 98 & $\|$ & $\|$ & $10 / 10$ & III primary school class \\
\hline & 23 & M & 7,1 & 34 & 1860 & 72 & $\|$ & III & $7 / 10$ & I primary school class \\
\hline & 24 & $\mathrm{~F}$ & 7,2 & 32 & 1600 & 71 & $\|$ & II & $10 / 10$ & II primary school class \\
\hline & 25 & M & 7,8 & 30 & 1350 & 78 & IV & III & $9 / 10$ & Il primary school class \\
\hline \multicolumn{11}{|l|}{ Group } \\
\hline & 26 & $\mathrm{~F}$ & 9,3 & 32 & 1560 & 100 & $\|$ & $\|$ & $10 / 10$ & IV primary school class \\
\hline & 27 & $\mathrm{~F}$ & 10,11 & 26 & 1090 & NA & $\|$ & II & $6 / 10$ & $\mathrm{~V}$ primary school class \\
\hline & 28 & $M$ & 10,5 & 31 & 1900 & 96 & III & III & $10 / 10$ & V primary school class \\
\hline & 29 & M & 9,2 & 32 & 1600 & NA & I & I & $10 / 10$ & IV primary school class \\
\hline & 30 & M & 9,8 & 31 & 1390 & 112 & II & i & $6 / 10$ & IV primary school class \\
\hline
\end{tabular}

Note: GA, gestational age; Verbal Quotients: Verbal IQ on WISC- III, WPPSI-III, or Verbal Comprehension Index on WISC-IV; NA: not available. MACS levels: Manual Ability Classification System; GMFCS levels: Gross Motor Function Classification Systems.

means of the WPPSI subtest "Picture naming" to make sure they would be able to carry out the tasks of the battery (based on a verbal response). The characteristics of the TD sample are reported in Table 2 .

Exclusion criteria for both groups were: i) Retinopathy of Premature at birth more than II stage, ii) epilepsy not under pharmacological control, iii) learning disabilities, iv) behavioural disorders.

\section{Experimental design}

The experimental design was the same as that published by Purpura and colleagues, with data from typical pre-school and school-aged children (Purpura et al., 2018). Thirty common objects were selected from those which are typically used in the daily life of children in the selected age range (see Table 3). The objects were randomized into three 
Table 2. The distribution of TD children by age group.

\begin{tabular}{|c|c|c|c|}
\hline $\begin{array}{l}\text { Group A } \\
n=37\end{array}$ & $\begin{array}{l}\text { II kindergarten class } \\
\text { Average age } 4.7 \mathrm{ys} \\
\text { Mean RPM } 67.94^{\text {th }} \text { percentile } \\
\text { III kindergarten class } \\
\text { Average age } 5.6 \mathrm{ys} \\
\text { Mean RPM } 80.21^{\text {th }} \text { percentile }\end{array}$ & 11 males & 9 females \\
\hline $\begin{array}{l}\text { Group B } \\
n=40\end{array}$ & $\begin{array}{l}\text { I primary school class } \\
\text { Average age } 6.3 \mathrm{ys} \\
\text { Mean RPM } 86.91^{\text {th }} \text { percentile } \\
\text { II primary school class } \\
\text { Average age } 7.3 \mathrm{ys} \\
\text { Mean RPM } 55.93^{\text {th }} \text { percentile } \\
\text { III primary school class } \\
\text { Average age } 8.2 \mathrm{ys} \\
\text { Mean RPM } 68.15^{\text {th }} \text { percentile }\end{array}$ & 4 males & 8 females \\
\hline $\begin{array}{l}\text { Group C } \\
n=39\end{array}$ & $\begin{array}{l}\text { IV primary school class } \\
\text { Average age } 9.4 \text { ys } \\
\text { Mean RPM } 54.23^{\text {th }} \text { percentile } \\
\text { V primary school class } \\
\text { Average age } 10.4 \text { ys } \\
\text { Mean RPM } 54.88^{\text {th }} \text { percentile }\end{array}$ & 13 males & 8 females \\
\hline
\end{tabular}

sets of 10 objects each. Children underwent the administration of all three sets, each assigned to one sensory modality, following pseudorandom criteria. In order to ensure that each set was equally presented in the visual, haptic, and visuo-haptic modality, six combinations of sets were prepared for administration to children (see Table 4).

Selection of the 30 objects was carried out also on the basis of size, to ensure that the children could grasp and manipulate all the objects easily with one hand.

Before administering the study protocol, we obtained written informed consent to participate in the study from the parents of each child. For every item, a time limit of 6 seconds was set, and we always encouraged the children to give an answer, even when they found the task difficult or were reluctant to form a hypothesis. As we had observed

Table 3. A schematic view of the 30 objects, divided into three sets.

\begin{tabular}{lll}
\hline SET A & \multicolumn{1}{c}{ SET B } & \multicolumn{1}{c}{ SET C } \\
\hline Ring & Coffee cup & Hair elastic \\
Die & Little sponge & Button \\
Little ball & Eraser & Screw \\
Dummy & Paper clip & Comb \\
Teaspoon & Little toy car & Little brush \\
Clothes peg & Bracelet & Battery \\
Coin & Key & Pen cap \\
Little candle & Little tube for toothpaste & Pencil sharpener \\
Cork (bottle cap) & Little toy bear & Building block (Lego-like) \\
Little dessert fork & Pencil & Children's scissors \\
\hline
\end{tabular}

Table 4. A schematic view of the six combinations of sets.

\begin{tabular}{lcccccc}
\hline SENSORY & COMBINATION & COMBINATION & COMBINATION & COMBINATION & COMBINATION & COMBINATION \\
MODALITY & 1 & 2 & 3 & 4 & 5 & 6 \\
\hline VISUAL & SET A & SET A & SET B & SET C & SET B & SET C \\
HAPTIC & SET B & SET C & SET C & SET B & SET A & SET A \\
VISUO-HAPTIC & SET C & SET B & SET A & SET A & SET C & SET B \\
\hline
\end{tabular}


in our previous study (Purpura et al., 2018), the protocol was easy and rapid to administer (15-20 minutes); the stimuli aroused the interest of the children, and this helped to hold their attention, guaranteeing a good level of compliance. We also included children who were not able to indicate the right name of the object but only its exact function. Children with refractive errors used their usual prescription lenses. In a previously published paper (Purpura et al., 2018), no evidence of significant correlations between correct answers and cognitive abilities in typical children was found.

\section{Visual object recognition}

In the visual object recognition task (V-ORT) children were asked to recognize the real object depicted in black-and-white photographs viewed from usual perspectives (see Figure 1). The photographs were all $12 \times 9.2 \mathrm{~cm}$. We decided to use photographs instead of drawings because photographs reproduce the three-dimensionality of objects more accurately, and we preferred high-contrast black-and-white photographs to minimize the facilitation of colour information on object individuation. Each photograph was removed after six seconds. The participants had to say the name of the object they had seen.

\section{Haptic object recognition}

The haptic object recognition task (H-ORT) consisted in the recognition of real objects through haptic exploration alone, thus without visual support. The experimenter put a real object into the dominant hand of the participant, inside a box which blocked the
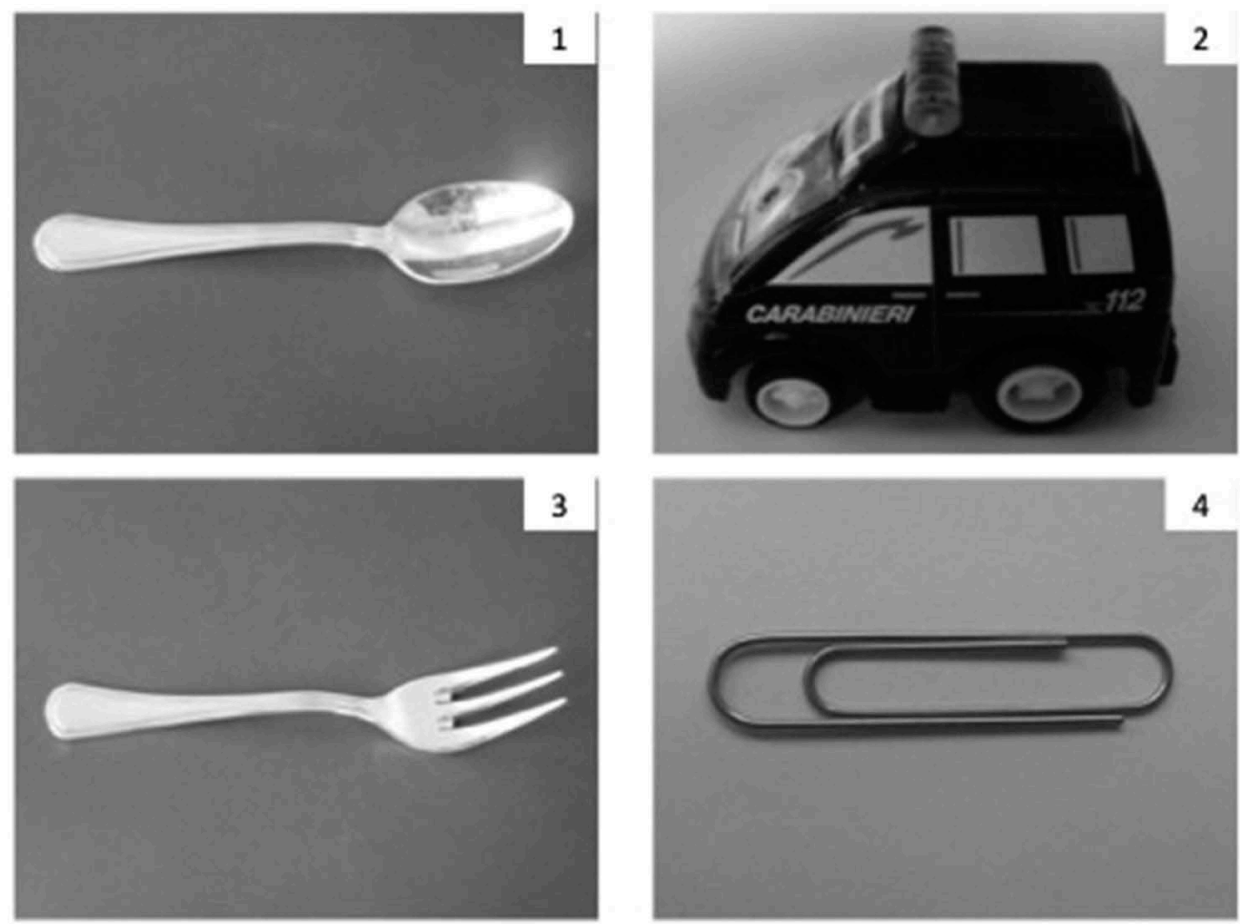

Figure 1. Examples of the stimuli used for the recognition tasks. 
participant's view, and asked him to say the name of the object. In each trial the object was removed after six seconds.

\section{Visuo-haptic object recognition}

The visuo-haptic object recognition task (VH-ORT) required the simultaneous use of the two sensory systems. In this case, children explored the object placed within a box touching it with their dominant hand, in accordance with the protocol of the Haptic Battery, but at the same time they were offered visual information too. The visual clue was represented by four high-contrast black-and-white photographs (size $12 \times 9.2 \mathrm{~cm}$ ) of 4 different objects (including the one inside the box). Three distractor stimuli were selected from objects similar in form or semantic category. The child had to say the name of the object that he was touching, choosing from the 4 objects that he could see in the photographs.

\section{Statistical analysis}

All analyses were achieved using SPSS 20.0.0. software. A p-value below 0.05 was interpreted as significant.

A mixed-design ANOVA analysis was carried out, with sensory modalities (V-ORT, $\mathrm{H}-\mathrm{ORT}, \mathrm{VH}-\mathrm{ORT}$ ) as a repeated-measure factor, and group (PVL and Typical) as a between-participant factor. Post-hoc tests (Bonferroni) were performed. The MannWhitney Test was used to compare the results of the experimental battery in the two groups of children (PVL and TD). Initially, the PVL group was compared with the entire control sample (30 PVL vs 116 TD) and then, by matching for age and gender 2 control children with every PVL patient (30 PVL vs 60 matched TD). Subsequently, the Mann-Whitney Test was also performed to compare PVL and TD across three age groups (Group A: second and third kindergarten classes; Group B: first, second, and third primary school classes; Group C: fourth and fifth primary school classes).

The Friedman Test and Wilcoxon Test were performed to compare V-ORT, H-ORT, and VH-ORT scores within the PVL group. Finally, a number of possible confounding factors were considered, in order to control for any kind of influence. Thus, for the PVL Group, a two-tailed bivariate non-parametric correlation test (Spearman Test) was performed between the different tasks and some of the cohort data (cognitive level, gestational age, birthweight, visual acuity, GMFCS levels and MACS levels). The Friedman test and Wilcoxon Test were also used to compare the distribution of motor impairment (GMFCS levels) and visual deficits (in particular visual acuity) across the three age groups of PVL children. Differences according to gender were analyzed by means of a parametric t-test.

\section{Results}

Mean values of correct answers across the two groups (PVL Group and TD Group) are reported in Figure 2 (PVL Group = Mean V-ORT: 7.26, SD: 2.3; Mean H-ORT: 5.66, SD 2.0; Mean VH-ORT: 8.23, SD 1.8; TD Group = Mean V-ORT: 8.83, SD = 0.9; Mean H-ORT: 7.86, SD = 1.7; Mean VH-ORT: 9.39, SD =1.1), showing better results in the visuo-haptic modality compared to the unisensory modalities in both groups; as regards 


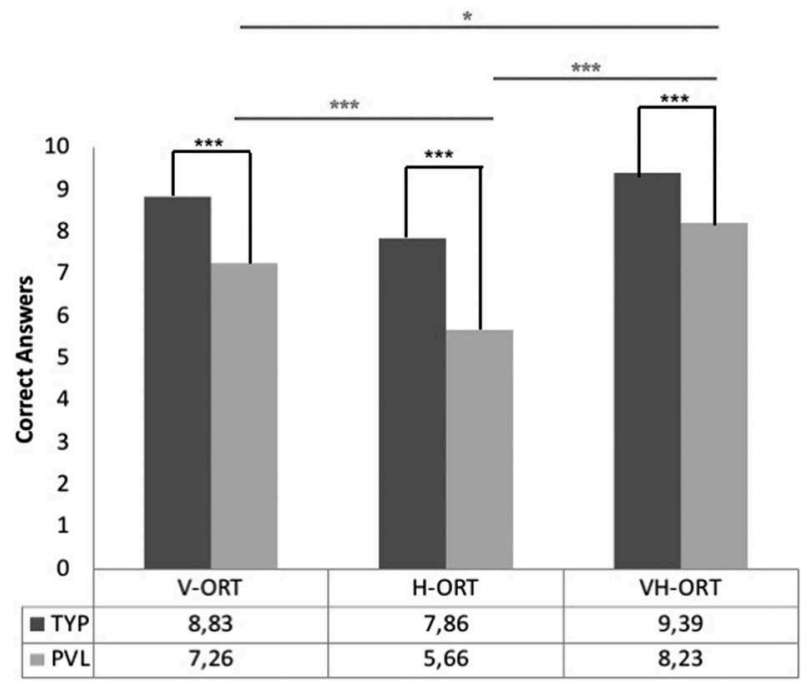

Figure 2. Mean of correct answers in the three sensory conditions across the two samples (PVL and TD groups). The grey lines and asterisks indicate the differences between the three sensory conditions in the PVL group. The asterisks indicate a significant difference between conditions: ${ }^{*} p \leq 0.05,{ }^{* *} p \leq 0.01,{ }^{* * *} p \leq 0.005$.

unisensory modalities, children reported a higher number of correct answers in the V-ORT compared to the H-ORT, both in the experimental group and in the control group.

A mixed-design ANOVA analysis with sensory modalities (V-ORT, H-ORT, VHORT) as a repeated-measure factor and group (PVL and TD) as a between-participant factor yielded a significant effect of sensory modalities $(\mathrm{F}=57.438, \mathrm{p}=<.001)$ and of group $(\mathrm{F}=55.128, \mathrm{p}=<.001)$ on task performance. These main effects were qualified by a significant interaction between sensory modalities and group $(\mathrm{F}=3.662, \mathrm{p}=.028)$. As indicated in Figure 2, comparisons between the two groups were significantly different across all the tasks (V-ORT: $\mathrm{p}=.001$; H-ORT $\mathrm{p}=<.001$; VH-ORT: $\mathrm{p}=.002$; Bonferroni corrected $\alpha=0.016[.05 / 3]$, independent sample t-test).

Results were also confirmed by non-parametric Mann-Whitney Test, because of the PVL group and the whole TD group showed statistically significant differences in correct answers $(\mathrm{p}=.001 ; \mathrm{z}=-3,361 ; \mathrm{p}=<.001 ; \mathrm{z}=-5,100 ; \mathrm{p}=<.001 ; \mathrm{z}=-3,894$; for V-ORT, H-ORT and H-ORT respectively, Bonferroni corrected $\alpha=0.016[.05 / 3]$ ). These results were confirmed in all three sensory modalities, even when the MannWhitney Test was performed comparing the PVL group with a smaller control group matched for age and gender (V-ORT: $\mathrm{p}=.006, \mathrm{z}=-2.767 ; \mathrm{H}-\mathrm{ORT}: \mathrm{p}=<.001$; $\mathrm{z}=-4.489 ;$ VH-ORT: $\mathrm{p}=<.001, \mathrm{z}=-3421)$.

On the basis of the three age groups (as reported in a previous paper by Purpura et al., 2018), it is possible to observe a different trend of development in unisensory and multisensory modality in PVL children, compared to TD children. As regards Group A, the Mann-Whitney Test showed a tendency to significance for V-ORT scores, a slight significance for H-ORT scores and an important significance for VH-ORT scores (V-ORT: $\mathrm{p}=.074, \mathrm{z}=-1.786$; H-ORT: $\mathrm{p}=.015, \mathrm{z}=-2,434 ;$ VH-ORT: $\mathrm{p}=.001$, 
$\mathrm{z}=-3,242)$. As regards Group $\mathrm{B}$, the significance was present in all three sensory conditions (V-ORT: $\mathrm{p}=.002, \mathrm{z}=-3.171$; H-ORT: $\mathrm{p}=<.001, \mathrm{z}=-3,634$; VH-ORT: $\mathrm{p}=.007, \mathrm{z}=-2,684)$. Finally, in Group $\mathrm{C}$, the difference remained significant only for H-ORT (V-ORT: $\mathrm{p}=.780, \mathrm{z}=-.279$; H-ORT: $\mathrm{p}=.012, \mathrm{z}=-2.511 ; \mathrm{VH}-\mathrm{ORT}: \mathrm{p}=.868$, $\mathrm{z}=-1.66)$.

In Figure 3 we report the mean values of the correct answers in the three different sensory conditions across the three age groups, for the PVL Group and for the Control Group. A progressive improvement in the number of correct answers was evident as age increased in both the experimental and control groups.

To analyse the results of the whole sample of PVL children in greater detail, the Wilcoxon Test was used and revealed significant differences between correct answers at $\mathrm{V}-\mathrm{ORT}$ and correct answers at H-ORT $(\mathrm{p}=.003, \mathrm{z}=-2.988)$, between correct answers at V-ORT and correct answers at VH-ORT $(\mathrm{p}=.019, \mathrm{z}=-2.349)$, and between correct answers at H-ORT and correct answers at VH-ORT $(\mathrm{p}=<.001, \mathrm{z}=-4.405)$ (see Figure 2). Subsequently, we analysed the distribution of correct answers in the three sensory modalities across the three age groups (see Table 5). As regards Group A, the Friedman Test showed a lack of significant differences between the three sensory

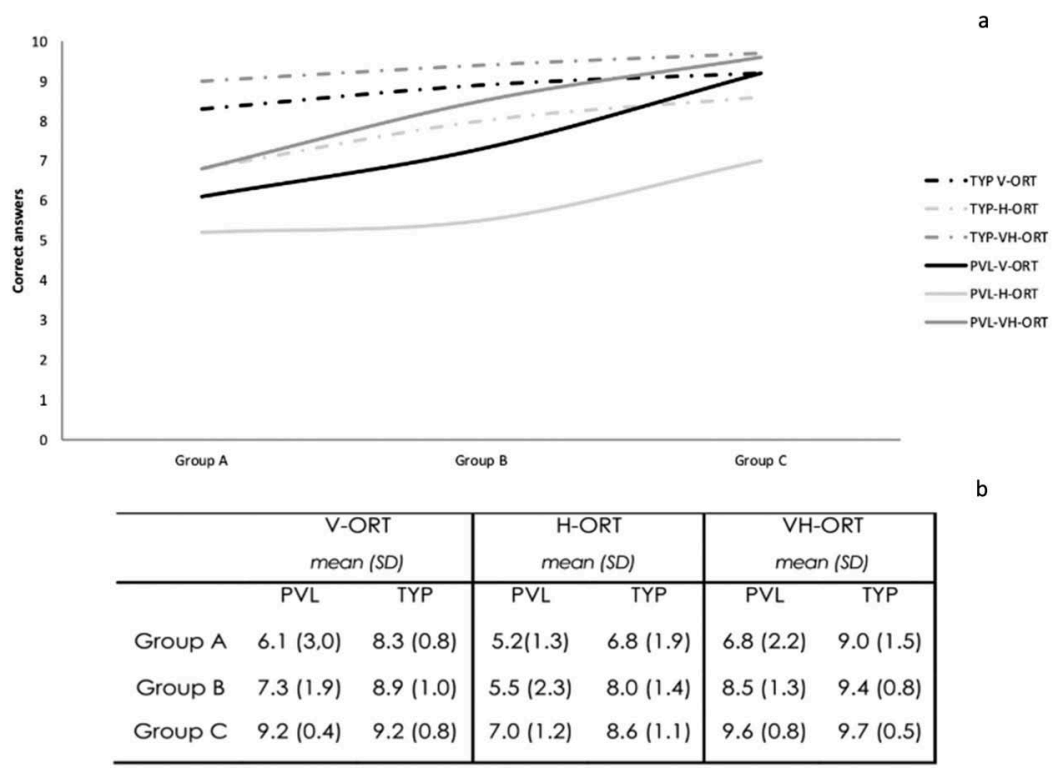

Figure 3. Developmental trend in the three sensory conditions both in the PVL group and in the TD group.

Table 5. Results from the Wilcoxon Tests and Friedman Tests on the PVL Group in the three sensory conditions across age groups.

\begin{tabular}{llll}
\hline & Group A & Group B & Group C \\
\hline V-ORT vs H-ORT & .336 & $\mathbf{. 0 1 5} *$ & .059 \\
V-ORT vs VH-ORT & .428 & $\mathbf{. 0 2 7} *$ & .317 \\
H-ORT vs VH-ORT & $\mathbf{. 0 2 7} *$ & $\mathbf{. 0 0 1} * *$ & $\mathbf{. 0 4 2} *$ \\
Friedman Tests & .159 & $\mathbf{. 0 0 0} * * *$ & $.024 *$ \\
\hline
\end{tabular}

The asterisks indicate a significant difference between conditions: ${ }^{* *} p \leq 0.05$, ${ }^{* *} \mathrm{p} \leq 0.01,{ }^{* * *} \mathrm{p} \leq 0.005$. 
conditions $\left(\mathrm{p}=.159, \chi^{2}=3.677\right)$ and the Wilcoxon Test detected a significant difference only between H-ORT and VH-ORT (V-ORT vs H-ORT: $\mathrm{p}=.336, \mathrm{z}=-.962$; V-ORT vs VH-ORT: $\mathrm{p}=.428, \mathrm{z}=-.704$; H-ORT vs VH-ORT: $\mathrm{p}=.027, \mathrm{z}=-2.209$ ). As regards Group B, the Friedman Test showed a highly significant difference between the three sensory conditions $\left(p=.000, \chi^{2}=16.262\right)$ and the Wilcoxon Test confirmed significant differences between all the conditions (V-ORT vs H-ORT: $\mathrm{p}=.015, \mathrm{z}=-2.427$; V-ORT vs VH-ORT: $\mathrm{p}=.027, \mathrm{z}=-2.213$; H-ORT vs VH-ORT: $\mathrm{p}=.001, \mathrm{z}=-3.254)$. As regards Group $\mathrm{C}$, the Friedman Test showed a significant difference between the three sensory conditions $\left(\mathrm{p}=.024, \chi^{2}=7.444\right)$ and the Wilcoxon Test specifically reported a significant difference between H-ORT and VH-ORT and a tendency to significance between V-ORT and H-ORT (V-ORT vs H-ORT: $\mathrm{p}=.059, \mathrm{z}=-1.890$; V-ORT vs VHORT: $\mathrm{p}=.317, \mathrm{z}=-1.000$; H-ORT vs VH-ORT: $\mathrm{p}=.042, \mathrm{z}=-2.032$ ).

For a more detailed description of findings with TD children see Purpura et al., 2018.

Results from Spearman's Correlation Test confirm the absence of significant correlations between correct answers in the three tasks and verbal cognitive abilities for the PVL Group, as already observed in TD children (Purpura et al., 2018). These tests were employed to check the potential effects of cognitive abilities on performance in experimental tasks and to verify the homogeneity of the sample. No differences in correct answers at V-ORT, H-ORT and VH-ORT were found when the PVL group was divided in two subgroups on the basis of the verbal IQ (the first group with verbal IQ from 70 to 90 , the second group with verbal IQ >90).

Moreover, no correlation was found between correct answers in the three tasks and gestational age or birth weight.

As expected, correlations were found between GMFCS and H-ORT scores, but also between visual acuity and V-ORT scores. As regards motor impairment, the Spearman Test showed a close link between GMFCS levels and H-ORT correct answers, while no correlations were found between GMFCS and V-ORT and VHORT (V-ORT: rho $-.174, \mathrm{p}=.356$; H-ORT: rho: $-.463, \mathrm{p}=.010$; VH-ORT: rho: $-.329, \mathrm{p}=.076)$. These data confirm the presence of a connection between the degree of motor impairment and manual exploration modalities. In fact, all PVL children had gross-motor impairment, with a GMFCS classification range from Level I (minor limitations) to Level IV (severe limitations). As regards MACS levels, a correlation was found with correct answers at VH-ORT (V-ORT: rho -.310, $\mathrm{p}=.096$; H-ORT: rho: $-.333, \mathrm{p}=.072$; VH-ORT: rho: $-.398, \mathrm{p}=.029$ ). According to these results, a more severe limitation in manual function correlates with a lower score in the cross-modal task. As regards visual acuity, it seemed to affect the V-ORT scores, but there were no correlations between visual acuity and H-ORT and VHORT (V-ORT: rho $-.459, \mathrm{p}=.011$; H-ORT: rho: $-.026, \mathrm{p}=.0892$; VH-ORT: rho: $-.725, \mathrm{p}=.070)$. It is important to note that sensorimotor deficits did not appear to influence visuo-haptic transfer. No significant correlations were found between GMFCS levels and visual acuity. No significant differences were detected between the three age groups in the distribution of GMFCS levels and visual acuity, so the degree of sensorimotor deficit was homogeneous in the three age bands.

As in typical children also in PVL group, males have worse thresholds besides females except for VH-ORT (Males: mean V-ORT: 6,85, SD = 2.37; mean H-ORT: $5.85, \mathrm{SD}=2.22$; mean VH-ORT: $8.38, \mathrm{SD}=1.65$; Females: mean V-ORT: $8.22, \mathrm{SD}=2.1$; 
mean H-ORT: $5.22, \mathrm{SD}=1.39$; mean VH-ORT: $7.88, \mathrm{SD}=2.26)$. In the all three tasks both males and females had worse scores compared to the control group: (Males: V-ORT $\mathrm{p}=<.001 ; \mathrm{t}=4.798, \mathrm{H}$-ORT: $\mathrm{p}=.002 ; \mathrm{t}=3.325$ and VH-ORT $\mathrm{p}=.044$; $\mathrm{t}=2.105 ;$ Females, V-ORT $\mathrm{p}=.038 ; \mathrm{t}=2.125 ; \mathrm{H}$-ORT $\mathrm{p}=<.001 ; \mathrm{t}=2.729 ;$ VH-ORT $\mathrm{p}=<.001 ; \mathrm{t}=4.774)$.

\section{Discussion}

The present results support the hypothesis that early visuo-haptic transfer is crucial for the normal development of object recognition across the life-span. This prediction was based on the finding of a previous study (Purpura et al., 2018), showing that visuohaptic transfer is already present in early childhood (4-5 years of age), but its maturation is very long and gradual during the life of individuals, continuing for the whole period of primary school. The current study tested a complementary prediction: patients with abnormal early experiences in visual and haptic perception should show a different trend of maturation in cross-modal calibration of visuo-haptic information for object recognition. To verify this hypothesis, we tested 30 children with PVL and bilateral cerebral palsy, as early unisensory and multisensory processing can be compromised in patients with complex and congenital disabilities.

The main result of this study is that, for all the sensory modalities investigated, there is an important difference between PVL and TD children, which indicates specific defects in the development of visual, haptic, and visuo-haptic recognition skills. In PVL participants as in TD participants, we found an age-dependent development of object recognition abilities for visual, haptic and visuo-haptic modalities, even if PVL children performed worse in all three sensory conditions, in comparison with controls, in particular until the third grade of primary school.

These results show specific deficits in visual and haptic unisensory processing in children with bilateral brain lesions and motor disorder, in line with findings by other research groups (Fazzi et al., 2009; Wingert et al., 2008). However, this study is, to our knowledge, the first one to analyse the evolution of transfer abilities between visual and haptic information in this population.

As regards unisensory processing, several difficulties were detected by the GMFCS and visual acuity assessments, suggesting that early perceptual-motor experiences play an important role in visual and haptic exploration of objects. Nevertheless, PVL children reported a higher number of correct answers in the visual modality versus the haptic one, considering the whole sample as well as the three age groups (see Figures 2 and 3). These data are very similar to those observed in TD children (Purpura et al., 2018). However, it is essential to consider that performance in the haptic recognition task was always significantly worse in the PVL group than in the TD group until the end of primary school, whereas, as regards the visual recognition task, the gap with TD children tended to decrease progressively and there was an important improvement from 9 years of age, despite there being no improvement in visual acuity. In fact, at this age visual abilities for object recognition appeared very similar to TD visual abilities, while PVL haptic processing remained compromised.

These results are in line with other findings concerning the development of visuocognitive abilities, which showed that "ventral stream" organization (responsible also 
for visual object recognition) is very long and complex and its development is strongly linked to daily life experiences in the social environment and consequently to the progressive emergence of gestalt perception (Bova et al., 2007; Fazzi et al., 2009). As a matter of fact, Bova and colleagues (Bova et al., 2007) highlighted that the typical maturation of the ventral processing stream continues for the majority of childhood (between 6 and 11 years of age) and the development of better and more efficient object recognition skills is connected to the capacity to retrieve visual information about daily experiences from memory.

Furthermore, in our experiment the visual task consisted in the recognition of blackand-white photographs of familiar objects, viewed from usual perspectives, while Fazzi and collaborators (Fazzi et al., 2009) suggested that in PVL children the impairment in visual recognition is specific to rotated objects in unusual perspectives and this deficit is explainable in terms of a malfunctioning of the dorsal stream as well as of the ventral stream. In this sense, the visual recognition difficulties of PVL children could be due to a dysfunction in the integration between the two pathways (ventral and dorsal), as the dorsal stream has an important role in the perception and in the understanding of the object's perspective. This assumption could explain the progressive amelioration in visual recognition skills in our task, which involves above all ventral stream abilities (perception and recognition of the shape of familiar non-rotated objects), although no difference in visual acuity was found between the three age groups. This finding is in line with data from a previous study, which showed that children with SD are more accurate in memorizing object form than spatial position (Di Lieto et al., 2017).

This explanation could also justify the difficulties in haptic processing in PVL children, which appeared stable and salient until the end of primary school (see Figure 3). Indeed, the dorsal stream mediates the visual-motor transformations required for object grasping and manipulation during daily life. In line with this assumption Wingert and colleagues (Wingert et al., 2008) found specific and bilateral deficits in roughness discrimination and tactile object recognition in children with diplegia and, more recently, Di Lieto and collaborators (Di Lieto et al., 2017) suggested that sensorymotor deficits in children with SD appear specific to higher-order sensory-motor representation, integration and planning, while coordination skills of distal upper limb movements may be relatively preserved.

On the other hand, as regards multisensory processing, visuo-haptic performance appeared more accurate than unisensory visual and haptic performance in PVL children, as in TD children (see Figure 3). It is also interesting to note how visuo-haptic transfer enhanced object recognition skills, despite the difficulties in unisensory processing. Specifically, visual acuity and motor impairment (measured with the GMFCS) were correlated respectively with visual and haptic tasks, but they did not seem to influence multisensory processes.

Visual recognition skills in PVL sample improved in a very rapid manner across age groups, leading to an increased use of this channel for object recognition, which, in typical children, is usually also guided by haptic information. While cross-modal visuohaptic recognition of familiar objects appears present, although not fully mature in typical pre-school aged children (Bushnell \& Baxt, 1999; Purpura et al., 2018), in our study, a different trend of maturation was found in PVL children. In particular, young children (4-5 years of age) did not exhibit the same levels of facilitation from cross- 
modal transfer compared to the controls of the same age (Purpura et al., 2018). As a matter of fact, in the object recognition task PVL children appeared to benefit only partially from cross-modal transfer abilities, having to compensate for the deficit related to haptic abilities.

As regards object recognition, it could be assumed that in PVL children, visual information becomes progressively more salient during development in comparison to tactile information. So, according to the principle of inverse effectiveness (Stein, Stanford, \& Rowland, 2014), multisensory processes cannot occur because visual information is stronger and more organized. So, during the cross-modal transfer task, the constituent unisensory stimuli cannot evoke a better response than that elicited by the visual task alone. Unfortunately, our findings cannot be compared with previous studies as no specific data are available in the literature on multisensory processes in children with PVL and cerebral palsy.

Another possible explanation may be linked to the progressive maturation of visuohaptic perception during childhood. Indeed, in the second part of the first year of life, infants begin to use haptic information to adjust their grip configuration to object size and texture before they begin to rely on visual information alone (Corbetta \& SnappChilds, 2009), but in children with PVL this kind of experience could be abnormal from the earliest stages of life, because the areas typically affected by this type of lesion are correlated with important difficulties in visual-motor integration abilities (Pagliano et al., 2007).

In conclusion, this study brings a novel contribution to the literature by suggesting the presence of a different trend of development in unisensory and multisensory skills for object recognition in children with PVL. Our findings show that PVL children have a specific deficit both in visual and haptic information processing that improves with age, probably thanks to everyday experience, but their sensory profile remains characterized by a deficit in haptic recognition skills and by an atypical cross-modal calibration for object recognition. Moreover, it could be fundamental for patients with complex neurodevelopmental disabilities, to analyse the interlink between the different sensory modalities in order to understand the learning mechanisms of these children better, and ultimately to develop new strategies for early rehabilitation.

\section{Acknowledgments}

The authors would like to thank Giada Frosini and Chiara Poli for their collaboration in the recruitment of children. We also thank Natasha Chericoni for reviewing the English of the manuscript.

\section{Disclosure statement}

No potential conflict of interest was reported by the authors.

\section{References}

Bax, M., Tydeman, C., \& Flodmark, O. (2006). Clinical and MRI correlates of cerebral palsy: The European Cerebral Palsy Study. JAMA, 296(13), 1602-1608. 
Bova, S. M., Fazzi, E., Giovenzana, A., Montomoli, C., Signorini, S. G., Zoppello, M., \& Lanzi, G. (2007). The development of visual object recognition in school-age children. Dev Neuropsychol, 31(1), 79-102.

Bushnell, E. W., \& Baxt, C. (1999). Children's haptic and cross-modal recognition with familiar and unfamiliar objects. J Exp Psychol Hum Percept Perform, 25(6), 1867-1881.

Corbetta, D., \& Snapp-Childs, W. (2009). Seeing and touching: The role of sensory-motor experience on the development of infant reaching. Infant Behav Dev, 32(1), 44-58.

Di Lieto, M. C., Brovedani, P., Pecini, C., Chilosi, A. M., Belmonti, V., Fabbro, F., ... Cioni, G. (2017). Spastic diplegia in preterm-born children: Executive function impairment and neuroanatomical correlates. Res Dev Disabil, 61, 116-126.

Eliasson, A. C., Krumlinde-Sundholm, L., Rosblad, B., Beckung, E., Arner, M., Ohrvall, A. M., \& Rosenbaum, P. (2006). The Manual Ability Classification System (MACS) for children with cerebral palsy: Scale development and evidence of validity and reliability. Dev Med Child Neurol, 48(7), 549-554.

Fazzi, E., Bova, S., Giovenzana, A., Signorini, S., Uggetti, C., \& Bianchi, P. (2009). Cognitive visual dysfunctions in preterm children with periventricular leukomalacia. Dev Med Child Neurol, 51(12), 974-981.

Gori, M., Del Viva, M., Sandini, G., \& Burr, D. C. (2008). Young children do not integrate visual and haptic form information. Curr Biol, 18(9), 694-698.

Gori, M., Sandini, G., Martinoli, C., \& Burr, D. (2010). Poor haptic orientation discrimination in nonsighted children may reflect disruption of cross-sensory calibration. Curr Biol, 20(3), 223-225.

Gori, M., Tinelli, F., Sandini, G., Cioni, G., \& Burr, D. (2012). Impaired visual size-discrimination in children with movement disorders. Neuropsychologia, 50(8), 1838-1843.

Joanne Jao, R., James, T. W., \& Harman James, K. (2014). Multisensory convergence of visual and haptic object preference across development. Neuropsychologia, 56, 381-392.

Jovanovic, B., \& Drewing, K. (2014). The influence of intersensory discrepancy on visuo-haptic integration is similar in 6-year-old children and adults. Front Psychol, 5, 57.

Lacey, S., \& Sathian, K. (2014). Visuo-haptic multisensory object recognition, categorization, and representation. Front Psychol, 5, 730.

Morrongiello, B. A., Humphrey, G. K., Timney, B., Choi, J., \& Rocca, P. T. (1994). Tactual object exploration and recognition in blind and sighted children. Perception, 23(7), 833-848.

Pagliano, E., Fedrizzi, E., Erbetta, A., Bulgheroni, S., Solari, A., Bono, R., ... Riva, D. (2007). Cognitive profiles and visuoperceptual abilities in preterm and term spastic diplegic children with periventricular leukomalacia. J Child Neurol, 22(3), 282-288.

Palisano, R., Rosenbaum, P., Walter, S., Russell, D., Wood, E., \& Galuppi, B. (1997). Development and reliability of a system to classify gross motor function in children with cerebral palsy. Dev Med Child Neurol, 39(4), 214-223.

Purpura, G., Cioni, G., \& Tinelli, F. (2018). Development of visuo-haptic transfer for object recognition in typical preschool and school-aged children. Child Neuropsychol, 24(5), 657-670.

Stein, B. E., Stanford, T. R., \& Rowland, B. A. (2014). Development of multisensory integration from the perspective of the individual neuron. Nat Rev Neurosci, 15(8), 520-535.

Volpe, J. J. (2003). Cerebral white matter injury of the premature infant-more common than you think. Pediatrics, 112(1 Pt 1), 176-180.

Volpe, J. J. (2009). Brain injury in premature infants: A complex amalgam of destructive and developmental disturbances. Lancet Neurol, 8(1), 110-124.

Wingert, J. R., Burton, H., Sinclair, R. J., Brunstrom, J. E., \& Damiano, D. L. (2008). Tactile sensory abilities in cerebral palsy: Deficits in roughness and object discrimination. Dev Med Child Neurol, 50(11), 832-838. 